\title{
Instituto Nacional de Enfermedades Respiratorias Ismael Cosío Villegas. Proyección y perspectiva. A 85 años
}

\author{
Instituto Nacional de Enfermedades Respiratorias Ismael \\ Cosío Villegas. Projection and perspective. At 85 years
}

\author{
José Luis Sandoval-Gutiérrez*
}

*Instituto Nacional de Enfermedades Respiratorias Ismael Cosío Villegas. Ciudad de México, México.

Palabras clave: Neumología, tuberculosis, enfermedades respiratorias. Keywords: Pneumology, tuberculosis, respiratory diseases.

Continuar la historia de una Institución no representa mera rutina. Conlleva un significado más trascendente: la captación de hechos de avance o transmutación, del recibo de impulsos y sofocamiento de defectos. Enrique Cárdenas de la Peña (febrero, 1998)

El Instituto Nacional de Enfermedades Respiratorias Ismael Cosío Villegas (INER), en su existencia mayor de ocho décadas, ha tenido una historia respiratoria de relevancia nacional e internacional. ${ }^{1,2}$

\section{PROYECCIÓN (2008-2015)}

En 2008 se nombra al Dr. José Rogelio Pérez Padilla como director general, iniciando un nuevo ciclo de apoyo a la investigación y aspectos académicos.

La pandemia de influenza A H1N1, en 2009, provocó el surgimiento de los primeros casos y muertes en nuestro instituto, las miradas del mundo se dirigieron a nuestro hospital, hubo la necesidad de reconvertir áreas para dar manejo oportuno a los pacientes aquejados con este virus,

Correspondencia:

Dr. José Luis Sandoval-Gutiérrez

Instituto Nacional de Enfermedades Respiratorias Ismael Cosío

Villegas.

Correo electrónico: sandovalgutierrez@gmail.com

Recibido: 28-V-2021; aceptado: 10-VI-2021.

Citar como: Sandoval-Gutiérrez JL. Instituto Nacional de Enfermedades Respiratorias Ismael Cosío Villegas. Proyección y perspectiva. A 85 años. Neumol Cir Torax. 2021; 80 (4): 286-290. https://dx.doi.org/10.35366/103453 la mitad del hospital se ocupó con este diagnóstico, las consultas se incrementaron exponencialmente al igual que la demanda de servicios y tratamiento antiviral.

Se colabora con instituciones nacionales e internacionales; tuvimos la visita de las diferentes agencias internacionales de salud como la Organización Panamericana de la Salud (OMS/OPS) y Center of Diseases Control (CDC), además de diferentes observadores extranjeros, todos reconocieron el enorme trabajo y desempeño del personal ante esta nueva enfermedad. Se adquirieron nuevos monitores y ventiladores de última generación para afrontar esta pandemia, hubo capacitación a médicos de primer contacto y se impartieron cursos online sobre esta enfermedad.

Se editó un libro sobre este tópico junto con la Sociedad Mexicana de Neumología y Cirugía de Tórax (SMNYCT) y se reportaron a través de artículos científicos los pormenores de la identificación, comportamiento y manejo de este virus (Figura 1).

La página web del instituto se ubicó, dentro de la red de hospitales a nivel mundial, como la más visitada en 2009.

Se recibió una comisión médica de Singapur interesados en el cultivo y manipulación del virus de la influenza A H1N1 teniendo una comunicación directa con la Dra. Manjarrez.

Hubo una producción científica importante sobre este tópico, se nombró al personal del INER como consultores de la OMS/OPS en influenza.

Se inició el plan de reconversión hospitalaria donde áreas de hospitalización se adecuaron para la atención del paciente crítico, llegándose a tener hasta 70 pacientes con intubación orotraqueal a la vez, el mismo esquema se utiliza en los años subsecuentes para las temporadas invernales de influenza anual.

Se impulsa la ventilación mecánica con la adquisición de equipamiento acorde a las necesidades del paciente 
crítico y la preparación en aerosolterapia e inhaloterapia con cursos académicos anuales; se apertura la convocatoria del grado de Técnico Superior Universitario (TUS) en terapia respiratoria.

Se forma con personal del INER y de otros centros académicos la Asociación Mexicana de Bioseguridad (AMEXBIO), la cual tuvo gran relevancia en las cuestiones de control de infecciones y diseminación de la influenza A H1N1.

Se realizaron varios documentales por televisoras nacionales y extranjeras sobre cómo el instituto enfrentó la primera pandemia del siglo XXI.

Se logra la certificación hospitalaria acorde a los lineamientos del Consejo Nacional de Salud, basados en lo estipulado en la Joint Commission de Estados Unidos de América.

Aumenta el número de solicitantes a la escuela de enfermería para el grado de licenciatura, así como lo correspondiente al posgrado de terapia intensiva en enfermería.

Se realiza el Simposio Internacional de Bioseguridad y Biocustodia en las instalaciones del INER teniendo una concurrencia exitosa.

Se incrementa el número de rotaciones de médicos en proceso de formación de especialidad de diferentes sedes hospitalarias en el país.

Se remodela el Servicio de Urgencias Respiratorias, así como recuperación del Servicio de Cirugía.

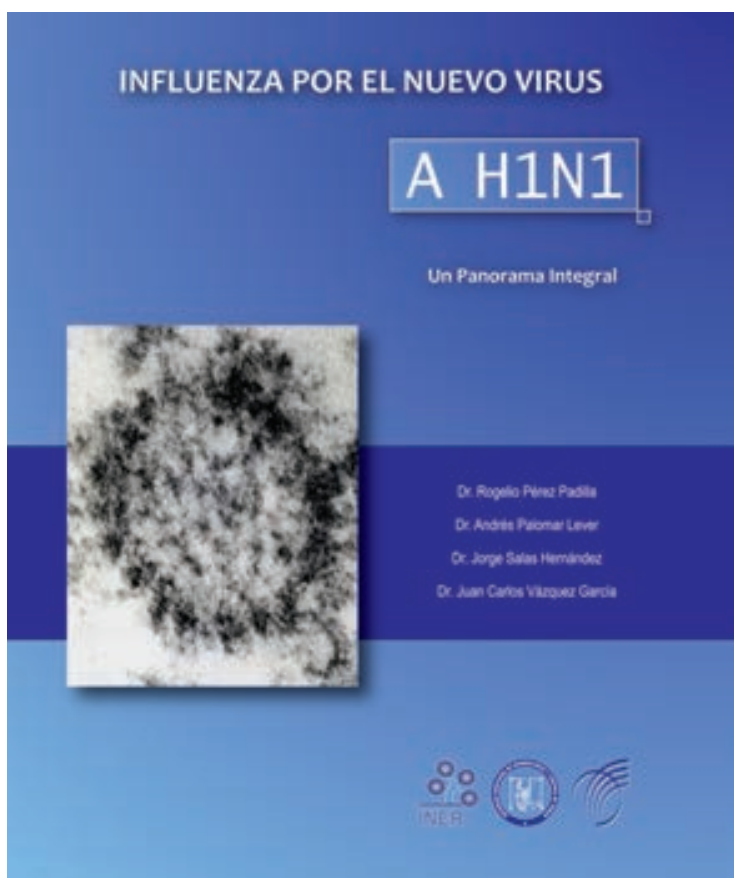

Figura 1: Libro Influenza por el nuevo virus A H1N1, editado por el Instituto Nacional de Enfermedades Respiratorias (INER) y la Sociedad Mexicana de Neumología y Cirugía de Tórax (SMNYCT).

Tomada de: https://www.laleo.com/influenza-por-el-nuevo-virus-a-h1n1-unpanorama-integral-p-6796.html
Se hacen cursos de ventilación mecánica y terapia respiratoria para médicos, enfermeras, inhaloterapistas y personal afín.

Se impulsa la capacitación en reanimación cardiopulmonar básica y avanzada (ACLS) para todo el personal a cargo del paciente crítico.

Se estimulan diferentes alianzas con la industria farmacéutica para la investigación de varios medicamentos con acción en la vía aérea.

Se difunde una cultura de bioseguridad a través de la formación del comité y la necesidad de la autorización del mismo para la realización de los nuevos proyectos de investigación.

Se recibe una delegación de personal de salud de Argentina que acuden con la comisión de observar la operatividad de nuestro instituto.

Se desarrollan cursos de ultrasonido e imagen de tórax impartidos por radiólogos y neumólogos del INER.

El instituto ha tenido una participación relevante en las medidas a seguir en salud ambiental y control del aire.

Médicos en proceso de formación para la especialidad en neumología hacen rotaciones a centros académicos en el extranjero para fortalecer su entrenamiento.

Se realizan las caminatas anuales para el paciente diabético/hipertenso, además de las tradicionales para el paciente con EPOC, fibrosis pulmonar y aquellos con el hábito de tabaquismo.

Se apoya con el servicio de ambulancia del servicio de urgencias respiratorias para traslados interhospitalarios por situación de estudios diagnósticos o procedimientos terapéuticos, así como los que requieran por situación especial su egreso a domicilio, inclusive a estados circunvecinos a la Ciudad de México.

Se apertura el pasillo cultural donde cada mes, artistas del INER o fuera del mismo, presentan diferentes expresiones, manifestando sus inquietudes, deseos o su versión de la vida contemporánea.

\section{PERSPECTIVA (2016-ACTUALIDAD)}

Hay una cantidad enorme de logros a través de la historia del INER (Tabla 1) y varios médicos han encabezado la Dirección General (Tabla 2).

Son muchos los retos que actualmente enfrenta el instituto, la epidemiología hospitalaria cambió, ya que sólo $5 \%$ de los ingresos son por tuberculosis, el resto representa patología variada, encabezando asma, neumonías, enfermedad pulmonar obstructiva crónica (EPOC), cáncer pulmonar, síndrome de apnea obstructiva del sueño (SAOS), patología quirúrgica, fibrosis pulmonar, hipertensión arterial pulmonar (HAP), paciente crítico neumológico, etcétera. ${ }^{3,4}$

La salud pública ha girado hacia la neumología, ya que los padecimientos respiratorios son la primera causa de 
Tabla 1: Logros relevantes del Instituto Nacional de Enfermedades Respiratorias.

\begin{tabular}{|l|}
\hline Primera intubación orotraqueal en una lobectomía en México \\
\hline Primer laboratorio de fisiología respiratoria en México \\
\hline $\begin{array}{l}\text { Primeros estudios sobre la efectividad de la estreptomicina y } \\
\text { kanamicina }\end{array}$ \\
\hline $\begin{array}{l}\text { Primeros trasplantes pulmonares (adulto y pediátrico) en } \\
\text { Latinoamérica }\end{array}$ \\
\hline $\begin{array}{l}\text { Primer reporte de características clínicas de los pacientes con } \\
\text { influenza A H1N1 }\end{array}$ \\
\hline Primera aplicación de la ventilación NAVA en México \\
\hline $\begin{array}{l}\text { Centro de investigación con más publicaciones internacionales de } \\
\text { neumopatía intersticial en el orbe }\end{array}$ \\
\hline Liderazgo en clínica de tabaquismo \\
\hline $\begin{array}{l}\text { Centro especializado en enfermedades respiratorias del paciente } \\
\text { con VIH }\end{array}$ \\
\hline $\begin{array}{l}\text { Primer caso detectado en México de COVID-19 (27 de febrero de } \\
2020)\end{array}$ \\
\hline
\end{tabular}

$\mathrm{NAVA}=$ neurally adjusted ventilatory assist; $\mathrm{VIH}=$ virus de la inmunodeficiencia humana.

muerte en el mundo y en México. ${ }^{5}$ La EPOC representa la tercera causa de muerte en el mundo. ${ }^{6}$

Se remodela el estacionamiento utilizando material que evita inundación, colocándose señales de protección civil para puntos de reunión y rutas de evacuación.

Se remodela el servicio de mantenimiento y servicios generales.

El instituto se ha convertido en un centro de referencia para el resto del país, tiene una actividad académica amplia y con alcance internacional.

Ha aumentado el número de aspirantes a la residencia médica de Neumología tanto del adulto como del paciente pediátrico, los cursos de cirugía de tórax y otorrinolaringología son galardonados como los mejores del país.

El Servicio de Inmunología y Alergia cuenta con una participación relevante dentro de la patología respiratoria, es común ver miembros de la comunidad médica participar y encabezar la Sociedad Mexicana de Neumología y Cirugía de Tórax (SMNYCT), el Consejo Nacional de Neumología $(\mathrm{CNN})$, participar en los congresos de ATS, la latinoamericana (ALAT) y la europea (ERS).

El personal sigue estando activo en los subsecuentes rebrotes de la influenza A H1N1, tuberculosis multidrogoresistente y en el paciente $\mathrm{VIH}$ con neumopatía.

El cáncer pulmonar tiene una atención especial en nuestro centro, aumentando los años de supervivencia de este mal, mejorando la atención y detección oportuna del mismo.
La mayoría de las escuelas de medicina tanto públicas como privadas en la Ciudad de México llevan su curso de neumología dentro de las instalaciones y cada vez más residentes de otras especialidades no neumológicas solicitan su rotación en nuestro instituto.

La mayoría de los egresados de la especialidad de neumología en el país se formaron en el INER.

El laboratorio de fisiología respiratoria es de los más relevantes en Latinoamérica, pionero de la capacitación en espirometría a través del curso avalado por la National Institute for Occupational Safety and Health (NIOSH) de los Estados Unidos.

Es el centro hospitalario con más neumólogos adscritos en el país y el principal lugar formador de cirujanos de tórax.

El Servicio de Rehabilitación Pulmonar es líder a nivel Latinoamérica, se tienen convenios con la fundación Teletón y con las diversas escuelas de medicina física y terapia respiratoria en el país.

El Departamento de Fibrobroncoscopia cuenta con el mayor número de procedimientos anuales en México.

Se remodela el Servicio de Laboratorio Clínico y se actualizan los dispositivos diagnósticos.

El INER es líder en el trasplante coclear contando con el apoyo de agrupaciones altruistas como Fundación Azteca para el desarrollo de los mismos.

La clínica de sueño es pionera en la formación de egresados de la especialidad, donde derivó la Academia Mexicana del Dormir.

Es centro de referencia nacional de tuberculosis multidrogorresistente.

Se cuenta con alrededor de 100 investigadores con reconocimiento en el Sistema Nacional de Investigadores (SNI), teniendo una productividad científica de más de 200 artículos indexados por año, convirtiéndonos en el tercer lugar dentro de los institutos nacionales de salud con atención asistencial.

Tabla 2: Directores generales del Instituto Nacional de Enfermedades Respiratorias.

\begin{tabular}{|l|}
\hline Dr. Donato G. Alarcón (1936-1947) \\
\hline Dr. Fernando Rébora Gutiérrez (1947-1956, 1965-1967) \\
\hline Dr. Ismael Cosío Villegas (1956-1965) \\
\hline Dr. Miguel Jiménez Sánchez (1967-1977) \\
\hline Dr. José Luis Luna Aguilar (1977-1980) \\
\hline Dr. Horacio Rubio Monteverde (1980-1992) \\
\hline Dr. Jaime Villalba Caloca (1993-2003) \\
\hline Dr. Fernando Cano Valle (2003-2008) \\
\hline Dr. José Rogelio Pérez Padilla (2008-2013) \\
\hline Dr. Jorge Salas Hernández (2013- ) \\
\hline
\end{tabular}


Se galardonó al Dr. Moisés Selman Lama con el Premio Nacional de Ciencias y Artes 2008, por sus grandes aportaciones a la patología intersticial pulmonar.

Varios miembros del INER pertenecen a la Academia Nacional de Medicina y en los últimos años se han anexado tres más, en la historia de la misma la han encabezado los Dres. Alarcón y Cosío, el primero además fue director de la Facultad de Medicina de la Universidad Nacional Autónoma de México (UNAM).

Existe intercambio académico con universidades del mayor prestigio fuera del país.

Se ha consolidado el desarrollo de maestrías y doctorados con reconocimiento de diferentes universidades, así como estancias posdoctorales de estudiantes nacionales y extranjeros.

Las escuelas de enfermería obtienen reconocimiento de la UNAM y la de terapia respiratoria con la titulación de técnico superior universitario, ésta es la primera en México y acaba de cumplir 12 años.

Se cuenta con la especialidad de Terapia Intensiva para licenciadas en enfermería con reconocimiento de la ENEO, teniendo en la actualidad más de 200 egresados.

Actualmente, el instituto atiende más de 16,000 urgencias al año, 4,500 ingresos, 70,000 consultas, cuenta con más de 130 médicos residentes nacionales y extranjeros de especialidad de diferentes áreas, casi 3,000 trabajadores en cuanto personal basificado y de contrato, 178 camas censables que pueden llegar a ser 230 con las no censables, lo cual nos convierte en el centro respiratorio más grande en el continente americano.

Se obtiene de nuevo la certificación hospitalaria por el consejo correspondiente.

Se impulsa el desarrollo de los diferentes modos ventilatorios y la utilización de la membrana de oxigenación extracorpórea (ECMO) en el paciente crítico.

Se produce la revista Neumología y Cirugía de Tórax (NCT) en convenio con la SMNYCT y seis asociaciones iberoamericanas.

En 2016 se otorga al INER el Premio Nacional de Calidad. ${ }^{7}$

El 22 de marzo de 2018 en el marco del LXXXII Aniversario del INER se rinde un homenaje póstumo al Dr. Donato G. Alarcón, reconociéndose con su nombre al edificio de gobierno (Figura 2). ${ }^{8}$

Es nombrado el Dr. Gustavo Reyes Terán médicoinvestigador del Centro de Investigación en Enfermedades Infecciosas (CIENI) del INER, titular de la Comisión Coordinadora de Institutos Nacionales de Salud y Hospitales de Alta Especialidad (CINSHAE).

El 27 de febrero de 2020 se diagnostica en el Servicio de Urgencias el primer paciente con SARS-CoV-2 virus agente del COVID-19 en el país, se intensifican las actividades de terapia intensiva a través de la reconversión

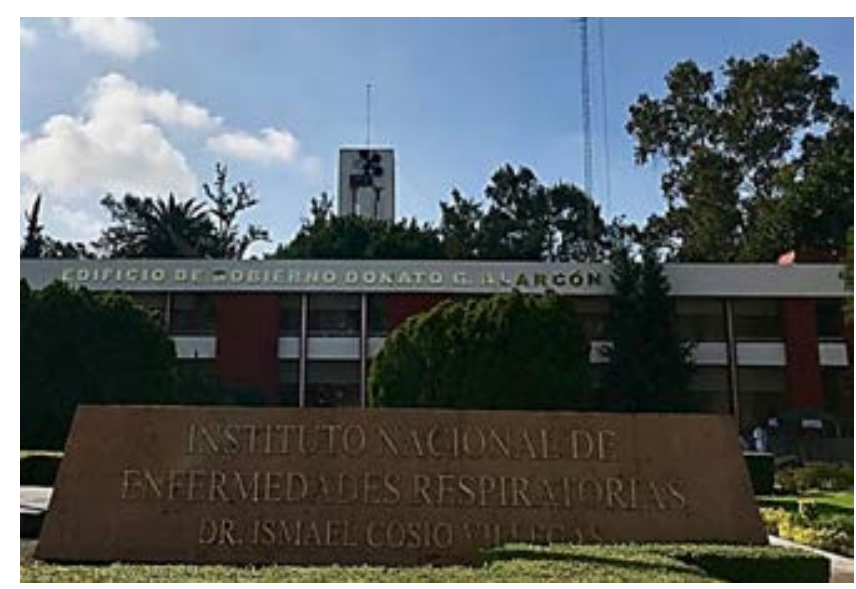

Figura 2: Edificio de Gobierno Donato G. Alarcón del Instituto Nacional de Enfermedades Respiratorias Ismael Cosío Villegas.

Tomada de: https://news-blogs.cisco.com/americas/es/2020/05/21/apoya-ciscoa-iner-con-soluciones-de-it-en-su-reconversion-hospitalaria-para-enfrentar-lacontingencia/

hospitalaria, se aprovecha la estructura de distanciamiento natural del edificio, Ilegando a tener más de 100 pacientes en promedio por día con intubación orotraqueal, ventilación mecánica y el manejo correspondiente de medicina crítica.

El INER se convierte en el hospital con más camas de terapia intensiva dedicadas al manejo de esta pandemia en Latinoamérica.

Se adquieren nuevos equipos de ventilación mecánica, ultrasonido, toma de placas portátiles de tórax y bombas de infusión.

Se autoriza por parte de la UNAM la apertura del Curso de Especialidad de Infectología de Adultos.

Se presenta el proyecto de especialidad dual de Neumología-Medicina Crítica.

Se impulsa la especialización de enfermería especialista en nefrología (UNAM-ENEO).

Se actualiza la página del instituto ofertándose la posibilidad de teleconsulta.

Se le otorga al INER el Premio Nacional de Salud por la iniciativa privada, también a nivel de la presidencia se reconoció con el galardón Miguel Hidalgo., ${ }^{910}$

Se termina la construcción de la nueva torre de investigación.

Se develan los murales de «Agradecimiento» $\mathrm{y}$ «Amigos del INER» a la entrada del Instituto con los nombres de las personas que aportaron su tiempo, conocimiento y esfuerzo a la lucha contra el COVID-19.

Como lo menciona el Dr. Rubén Sauceda Lumbres egresado del instituto en la década de los 90:

«El gigante añoso sigue dando batalla». 
Cada vez más miembros del ámbito académico, cultural, artístico, político y deportivo reconocen al INER como el lugar donde encontraron la salud de familiares, amigos o su persona.

La historia se está escribiendo, se ha avanzado a pasos agigantados en el último lustro. ${ }^{11}$

Para finalizar, el Dr. Enrique Cárdenas de la Peña mencionó sobre el INER:

«México entraña una ancestral tradición hospitalaria y el sanatorio Huipulco aparece como banderín de una pléyade de lugares que cura...»

Por todo lo anterior la nueva época está por escribirse y definirse en el futuro, caracterizándonos tres valores: orgullo, tradición y servicio.

iLarga vida a la salud respiratoria!

iLarga vida al INER!

\section{REFERENCIAS}

1. Instituto Nacional de Enfermedades Respiratorias. Ismael Cosío Villegas. [Accesado 15 de enero del 2021] Disponible en: https:// www.gob.mx/salud/

2. Cárdenas de la Peña E. Del Sanatorio de Huipulco al Instituto Nacional de Enfermedades Respiratorias. Cincuenta años. Volúmenes I-II. México: Secretaría de Salud; 1986.
3. Cárdenas de la Peña E. INER: cinco años más, 1987-1991. México, D.F.: SSA, INER; 1992.

4. Cárdenas de la Peña E. INER, 1992-1997 Continuidad. México: ECP; 1998.

5. Sandoval-Gutierrez JL, Reyes ES, Bautista EB. Pulmonary diseases: first cause of mortality in the world. Chest. 2011;139(6):1550. doi: 10.1378/chest.11-0021.

6. These are the top $10 \mathrm{global}$ causes of death - but two diseases are in decline. 2021 [Accesado 01 febrero del 2021]. Available in: https:// www.weforum.org/agenda/2021/02/top-10-global-causes-death/

7. Premio Nacional de Calidad en Salud 2016. [Accesado 01 de febrero de 2021] Disponible en: http://www.iner.salud.gob.mx/interna/ sistemacalidad_premionacionalcalidad2016.html

8. La Dirección General Informa. [Accesado 15 de febrero de 2021] Disponible en: http://iner.salud.gob.mx/descargas/educacion/boletin/ boletin109.pdf

9. El Premio Nacional de Salud un reconocimiento a la empatía y al amor en favor a México. [Accesado 03 de febrero de 2021] Disponible en: https://www.somoshermanos.mx/el-premio-nacional-de-salud-unreconocimiento-a-la-empatia-y-al-amor-en-favor-a-mexico/

10. Presidente entrega Condecoración 'Miguel Hidalgo' Grado Placa a hospitales federales COVID. [Accesado 17 de febrero 2021] Disponible en: https:/www.gob.mx/presidencia/prensa/presidenteentrega-condecoracion-miguel-hidalgo-grado-placa-a-hospitalesfederales-covid?idiom=es

11. Salas Hernández J. Olvera Masetto E. Instituto Nacional de Enfermedades Respiratorias Ismael Cosío Villegas. 80 años de servicio. México: Ed. Intersistemas; 2017.

Conflicto de intereses: El autor declara no tener conflicto de intereses. 